\title{
On the theory of induction-currents
}

\section{Mascart}

To cite this article: M. Mascart (1880) On the theory of induction-currents, Philosophical Magazine Series 5, 9:58, 452-455, DOI: 10.1080/14786448008626871

To link to this article: http://dx.doi.org/10.1080/14786448008626871

册 Published online: 28 Apr 2009.

Submit your article to this journal

Џ Article views: 2

Q View related articles $₫$ 
be produced, whereby the steady motion might be partially converted into vibratory motion. This effect would be precisely analogous to the generation by the siren of Cagniard de la Tour of musical sounds by means of a succession of interruptions of a steady current of air, the pitch of the sound increasing with the velocity of the current. These vibratory motions, being an accession to those produced by the speaker at the transmitting end of the telephone, and having a constant relation to them, are attended by an accession of current, and therefore an accession of effect at the receiving-end. This follows from the analytical formula in the second paragraph of the article in the Phil. Mag. for June 1878, the effects under consideration being all referable to pressures depending upon the squares of the velocity of a steady current of the ætherial medium.

I have now completed all that I am able to say relative to the department of physical science the principles of which were inaugurated by Newton, and which for distinction may be named Theoretical Physics. I must now leave to younger mathematicians the task of correcting and extending, if they think good, the efforts I have made in this direction, and shail only urge the important consideration that Theoretical Physics are as necessary for constituting a complete system of Physical Science as Experimental and Empirical Physics.

Cambridge, May 15, 1880.

\section{Intelligence and Miscellaneous Articles.}

ON THE THEORY OF INDUCTION-CURRENTS. BY M. MASCART.

M HELMHOLTZ has shown that, taking as starting-point the 1. laws of $\mathrm{Ohm}$ and Joule, the induction-phenomena produced by the displacement of a magnetic system in the vicinity of a current might, have been foreseen. It has seemed to me that a natural generalization of the results obtained in this particular case permits us to establish the theory of electrodynamic induction-currents so as to connect them simply with a common principle.

It is known that, according to Ampère's theory, the work necessary for displacing a magnetic mass $m$ in the vicinity of a current is equal to the product of that mass by the intensity $I$ of the current and by the increment of the angle under which the circuit is seen from two points occupied successively by the magnetic mass. The energy of the mass $m$ with respect to the current may therefore be represented by Imw. It will be useful to enunciate this property in another form by employing the notion of Faraday's lines of force.

If the force exerted by a magnetic system upon the unit of mass situated in a point be considered, and any surface-element whatever 
be carried through that point, the name stream of force (or number of lines of force) passing through that element may designate the product of its surface by the normal component of the force. It is readily seen that the stream of force of a mass $m$ in a cone of angular opening $\omega$ is equal to $m \omega$.

The energy of a magnetic mass with respect to a current is therefore equal to the product of the intensity by the stream of force emanating from that mass and passing through the circuit. Let us designate this stream of force by $\phi$, and agree to consider it positive when the forces enter the circuit through the negative surface of the current-that is, through the negative side of the equivalent magnetic plate; the energy of the mass $m$ will have for its expression

$$
\mathrm{I} m \omega=-\mathrm{I} \phi .
$$

Similarly, the energy with respect to a current of any magnetic system whatever is equal to the sum of the energies of all the masses - that is to say, to the product of the intensity of the current by the total stream of force of the system which passes through the circuit. This energy diminishes when the system is left to the action of the current; and for a slight displacement the work of the electromagnetic forces is equal to $+\mathrm{I} d \phi$.

If the displacement is effected during the time $d t$ and a liquidpile is employed, the energy derived from the chemical actions must heat the circuit and furnish the electrodynamic work Id $\phi$ corresponding to the increment $d_{\phi}$ of the stream of force, which gives, calling the electromotive force $E$ and the total resistance $R$,

$$
\mathrm{EI} d t=\mathrm{I}^{2} \mathrm{R} d t+\mathrm{I} d \phi, \ldots \ldots \ldots \ldots
$$

from which is deduced

$$
\mathrm{IR}=\mathrm{E}-\frac{d \phi}{d t} . \ldots \ldots \ldots \ldots \ldots \ldots
$$

The intensity of the current is the same as if there existed in the circuit a new electromotive force (induction) having the value

$$
e=-\frac{d \phi}{d t}
$$

The electromotive force of induction is therefore equal and of contrary sign to the derivate with respect to the time from the stream of force which emanates from the magnetic system and passes through the circuit. This expression, here deduced from the principle of the conservation of energy, is equivalent to that which Neumann obtained by starting from Lenz's law.

We shall assume as a general rule that the electromotive force of induction in a circuit is always expressed by equation (3) as a function of the stream of force which passes through it, whatever may be the canses which make the forces vary, such as the displacement or modification of a magnetic system, change of form, intensity, or position of an exterior current, deformation of the circuit itself, or variation of the current already passing through it. 
Such a generalization can be justified in each particular case when it is admitted that the effect produced by a change in the magnets and currents is equivalent to that which would be obtained by bringing from an infinite distance a magnet or current equal to the given variation. The consideration of the streams of force only will appear especially legitimate if it be conceived that electric and magnetic actions are not really exerted at a distance, but are due to a modification of the intervening medium, characterized at every point by the direction and magnitude of the force.

Let us first consider the effect of a current upon itself. The stream of force which traverses the circuit, supposed invariable, is proportional to the intensity of the current, and can be represented by UI, the factor $U$ designating the stream of force corresponding to the unit of current. If the variation of the current be $d \mathrm{I}$, the variation of the stream of force is $\mathrm{U} d \mathbf{I}$, and the energy derived from the pile

$$
\mathrm{IU} d \mathrm{I}=d\left(\frac{\mathrm{UI}^{2}}{2}\right)
$$

As long as the current has not become constant a part of the energy of the pile is therefore employed to augment the term $\frac{\mathrm{UI}^{2}}{2}$, called the potential energy of the current. Suppose that there is in the vicinity a second current, of intensity $I^{\prime}$, in a circuit of constant form. The stream of force from the second current which traverses the circuit of the first is proportional to the intensity; it may be represented by VI'. The factor $V$ denotes the stream of force that emanates from either of the two circuits and traverses the other when the two currents have the same intensity equal to unity. The product $\mathrm{II}^{\prime} \mathrm{V}$ is called the relative potential energy of the two currents.

When the product $I^{\prime} V$, in consequence of a change of intensity or a displacement, varies by $d\left(I^{\prime} V\right)$, the energy derived from the pile of the first current is $I d\left(I^{\prime} V\right)$. Therefore, if the two modifications are simultaneous and are produced during the time $d t$, we have for the first circuit

$$
\mathrm{EI} d t=\mathrm{I}^{2} \mathrm{R} d t+d\left(\frac{\mathrm{UI}^{2}}{2}\right)+\mathrm{I} d\left(\mathrm{I}^{\prime} \mathrm{V}\right) ; \ldots \ldots
$$

and, in like manner, for the second,

By addition we get

$$
\mathrm{E}^{\prime} \mathrm{I}^{\prime} d t=\mathrm{I}^{\prime 2} \mathrm{R}^{\prime} d t+d\left(\frac{\mathrm{U}^{\prime} \mathrm{I}^{\prime 2}}{2}\right)+\mathrm{I}^{\prime} d(\mathrm{IV}) . \ldots \ldots
$$

$\left(\mathrm{EI}+\mathrm{E}^{\prime} \mathrm{I}^{\prime}\right) d t=\left(\mathrm{I}^{2} \mathrm{R}+\mathrm{I}^{\prime 2} \mathrm{R}^{\prime}\right) d t+a\left(\frac{\mathrm{UI}^{2}}{2}+\frac{\mathrm{U}^{\prime} \mathrm{I}^{\prime 2}}{2}+\mathrm{II} \mathrm{V}\right)+\mathrm{II}^{\prime} d \mathrm{~V}$.

This equation expresses that the energy furnished by the two piles during the time $d t$ is employed in heating the conductors, increasing the potential energy of each of the currents as well as their relative potential energy, and, lastly, in furnishing the electrodynamic work corresponding to the relative displacement. If the 
form of the circuits be altered, the variation of the stream of force emanating from one of the currents in the direction of the other is comprised in the terms $d\left(I^{\prime} V\right)$ and $d(I V)$; and in order to value the action of a circuit upon itself it suffices to regard the factor $U$ as variable. We thus find again the known equations of electrodynamic induction-currents.

The same mode of reasoning permits us to take account of the changes which have happened in exterior magnets, and of the magnetization produced by currents on masses of soft iron.-Comptes Rendus de l'Académie des Sciences, April 27, 1880, t. xc. pp.981-984.

EXPERTMENTAL RESEARCHES ON THE DECOMPOSITION OF SOME

EXPLOSIVES IN A CLOSED VESSEL; COMPOSITION OF THE GASES FORMED. BY MM. SARRAU AND VIEILLE.

These researches were undertaken for the purpose of determining the conditions of the employment of gun-cotton in mines.

since the important improvements introduced by $\mathbf{M r}$. Abel into the manufacture of gun-cotton, this explosive is prepared in homogeneous masses of determined form and density, it is kept without danger in the wet state, and its explosive force, comparable with that of dynamite, is much greater than that of gun-powder ; consequently its use in mines affords great advantages. It presents, however, an inconvenience in that its explosion produces mephitic gases injurious to the workmen in the galleries. In fact its decomposition gives rise to the formation of carbonic oxide. This can be obviated by adding to the gun-cotton an oxidizer, such as a nitrate.

In this memoir we make a comparative study of the products formed, the heat evolved, and the pressure developed by the explosion in a closed vessel,-1st, of pure gun-cotton*; 2 ndly, of a mixture of equal parts of gun-cotton and nitrate of potass; 3 rdly, of a mixture of 40 parts of gun-cotton and 60 of nitrate of ammonia ; 4thly, of nitroglycerine ; and, 5thly, of ordinary miningpowder $t$. We exhibit today the results of our study of the gases formed during the explosion in a closed ressel.

Some previous experiments having shown that the nature and the composition of the gases depend on the conditions of their production, and particularly on the pressure under which they are formed, we operated upon gases collected in a closed test-tube,

* The composition, by weight, of gun-cotton, deduced from organic analysis, and verified by elementary analysis of the products of decomposition, has led us to regard the products of the manufacture current at the Moulin-Blanc works as a mixture of 3 eq. of trinitrated and 1 eq. of binitrated cellulose-

$$
3\left[\mathrm{C}_{12} \mathrm{H}_{7}\left(\mathrm{NO}_{4}\right)_{3} \mathrm{O}_{10}\right]+\mathrm{C}_{12} \mathrm{H}_{8}\left(\mathrm{NO}_{4}\right)_{2} \mathrm{O}_{10}
$$

corresponding to the rough formula

$$
\mathrm{C}_{48} \mathrm{H}_{28} \mathrm{~N}_{12} \mathrm{O}_{81}
$$

$\dagger$ This powder consists, from its manufacture, of 62 parts saitpetre, 20 parts sulphur, and 18 charcoal. 